\title{
Crowd Density Estimation System for Al-Masjid Al-Haram
}

\author{
S. Eldursi, N. Alamoudi, F. Haron, F. Aljarbua and G. Albakri
}

\begin{abstract}
Estimating crowd occupancy is an important element in crowd management and safety. Crowding is greatly manifested in the two holy mosques of Mecca and Medina which makes them a constant source of study and concern. As a solution to this problem we developed a prototype of Al-Haram Crowd Guide System; a client-server crowd density estimation system which uses texture based methods to assist in the guiding and management of crowds in Al-Masjid Al-Haram. This is achieved through a visual representation of the crowd occupancy presented to the users in the form of a heat-map. The main contribution of our paper is two-fold: first we explore the features of the system and its main design aspects and phases. Second, we provide a brief comparison between two texture-based techniques used to estimate crowd density; Grey Level Co-occurrence Matrices and Local Binary Patterns. To classify the images, they were coupled with a Self-Organizing Map and a Support Vector Machine respectively. Finally, we show examples of our system in operation tested on sample data gathered from the Saie area in Al-Masjid Al-Haram. We conclude with a summary of our results and some future considerations for the continuation of our work.
\end{abstract}

Index Terms - crowd density estimation, texture based analysis, Local Binary Pattern

\section{INTRODUCTION}

Due to increasing population growth and urbanization, the study of the crowd phenomenon has gained increasing scientific interest from a number of perspectives. The importance of understanding crowd behaviour is evident in many applications such as crowd management, public space design and visual surveillance [1]. Manual monitoring of crowds through surveillance camera systems to detect situations requiring attention is a tedious task. There has been extensive scientific

Manuscript received November 14, 2016.

S. Eldursi was with the Department of Computer Science, College of Engineering and Computer Sciences, Taibah University, Madinah, Kingdom of Saudi Arabia.

N. Alamoudi was with the Department of Computer Science, College of Engineering and Computer Sciences, Taibah University, Madinah, Kingdom of Saudi Arabia. She is now with the Department of Computer Science, Community College of Bader, Taibah University, Madinah, Kingdom of Saudi Arabia.

F. Haron was with School of Computer Sciences, University Sains Malaysia. She is now with the Department of Computer Science, College of Engineering and Computer Sciences, Taibah University, Madinah, Kingdom of Saudi Arabia.

F. Aljarbua was with the Department of Computer Science, College of Engineering and Computer Sciences, Taibah University, Madinah, Kingdom of Saudi Arabia.

G. Albakri was with the Department of Computer Science, College of Engineering and Computer Sciences, Taibah University, Madinah, Kingdom of Saudi Arabia. research with the aim of automating this process using computer vision techniques.

One of the crowd density estimation systems that have been applied in the real world is ADVISOR [2]; a visual surveillance system developed for use in metro stations to assist in the early detection of dangerous situations and to help avert further escalation by issuing warnings for possible intervention. The system relies on computer vision techniques to estimate crowd density amongst a variety of other crowd monitoring tasks. ADVISOR is the first system to integrate the capabilities of people tracking, crowd monitoring and behaviour analysis. Another crowd density estimation system is PRISMATICA [3] which is a system that depends on sensing technologies, that aims to improve personal security in public transportation. PRISMATICA tool uses "intelligent devices", such as cameras, capable of processing/analysis. The system consists of a camera network; a number of intelligent cameras placed in different areas, connected with each other by a local network to exchange data between them. It provides tasks such as intrusion detection in forbidden areas, pedestrian counting, calculating the occupation rate in strategic areas, queue length measurements and counter-flow detection.

Existing crowd density estimation mobile apps can be divided into two categories: based on image analysis such as the London Crowd App [4], and based on statistics such as the City of London Police App [5]. The City of London Police App is an application used for displaying crowd density based on counting the number of people within a particular area in London. It asks the users to share their locational data, which it will use to track the users and count them whenever there is a crowd. Finally it displays the results as a heat map. While London Crowd App displays the crowd density based on image analysis in London city. It has the capability to capture images from the street and analyze them then display the results as a heat map. It can also allow the user to view real time images to help guide the users and spread awareness of crowded areas to avoid.

In the two holy mosques, numerous expansions have been made over the years to accommodate the growing number of pilgrims and relieve congestion. The latest expansion project of Al-Masjid Al-Haram by the late King Abdullah currently in progress involves the expansion of the Tawaf area; where visitors of the Mosque circulate the Holy Ka'bah. The Tawaf area is expected to tolerate a capacity of up to 105,000 person per hour [6]. These ventures aim towards the control and management of crowds through the optimization of the architectural design of the buildings of Al-Masjid Al-Nabawi and Al-Masjid Al-Haram. Although, our system prototype strives towards the same aim we take on a different approach 
which relies on spreading awareness throughout the occupants of Al-Haram and authorized personnel. This prototype exploits the existence of the CCTV system in the holy mosque from which it acquires the relevant, instantaneous crowd image data. Using one of the crowd density estimation methods the images are analyzed to gain an estimate of the level of crowd density. This data is then translated into a comprehensive heat-map that graphically represents crowd density in various areas of the mosque. The heat-map can be viewed through a mobile application for authorized users or viewed on a display screen within Al-Masjid Al-Haram. Users of this program can utilize this map to avoid crowds or redirect to less occupied areas.

\section{Methodology}

This section introduces the methodology which was followed during the stages of this study. Fig. 1 shows the system's main phases the most important of which are the feature extraction and the classification phases.

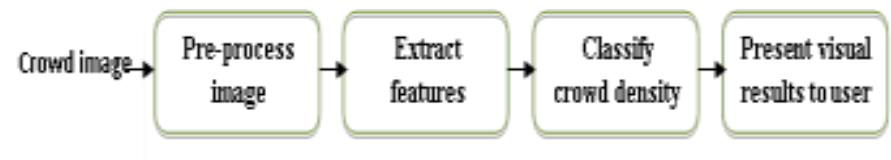

Fig. 1. Phases of the system

\section{A. System Architecture}

The context of the system as we view it is shown in Fig. 2. The system should reside within the control room server that receives information from Al-Masjid Al-Haram's CCTV system. The server proceeds to process the footage streaming from the CCTV system. The results can be viewed through a display screen in Al-Haram by visitors or accessed through a mobile app by the supervisors. System administrators control access to the application by registering the supervisors into the system with a username and password.

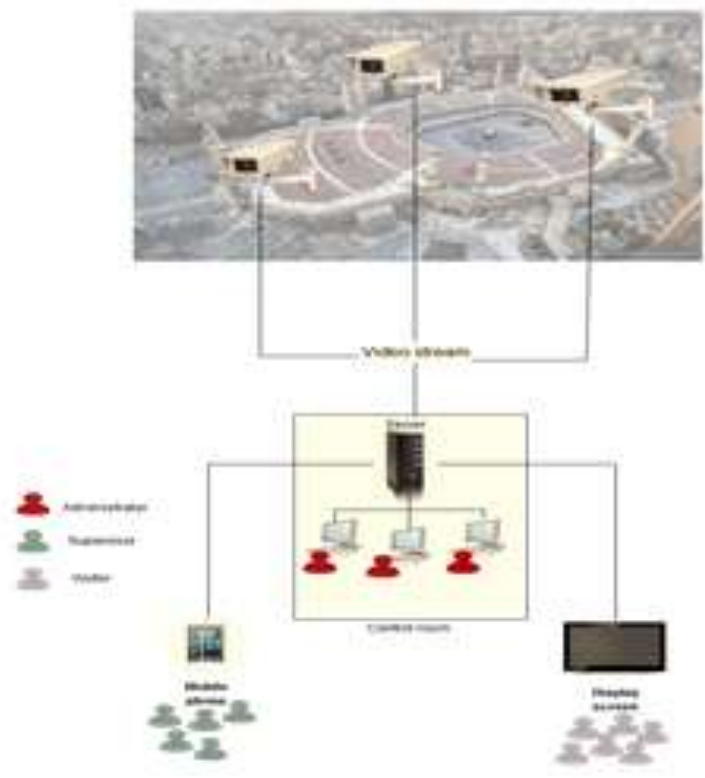

Fig. 2. System context
Fig. 3 presents a high level conceptual view of the system architecture. An explanation of each module's role is given below in the order of the flow of data through the system.

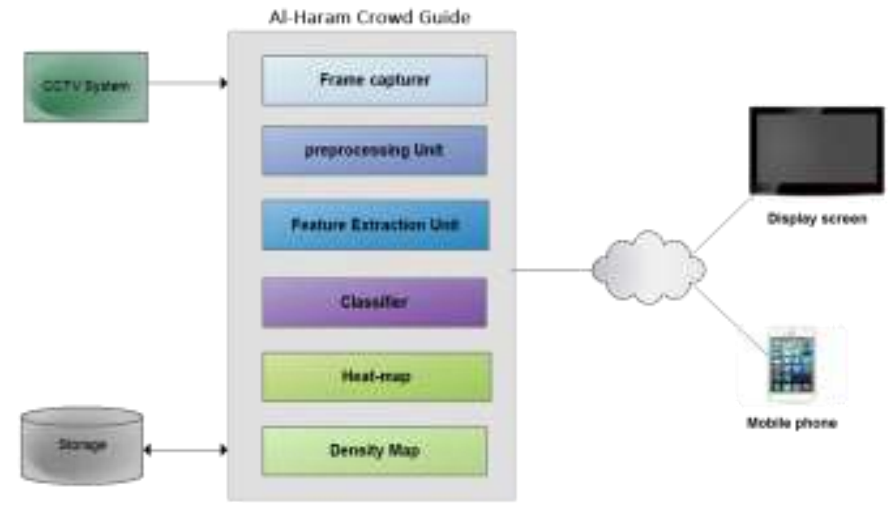

Fig. 3. System architecture and main system components

B. Modules and Sub-Modules of the System

1. Frame capturing module: Captures the frames from the video for processing.

2. Pre-processing module: performs the following processes on each image:

i. Converter to Grey scale: Converts the image into a grey scale and quantizes the number of grey-scales.

ii. Extract ROI (Region of Interest): Separates a specific part of the image.

iii. Enhance Image Contrast: Enhances the image contrast using histogram equalization

iv. Noise removal: Removes noise from the image using the median filter

v. Size normalization: Used to make all images the same size before processing.

vi. Perspective normalization: Used on tilted images to counteract the near-far effect.

3. Feature extraction module: Extracts texture features from the image for classification. In section 3 we compare two well-known methods of feature extraction, Grey Level Co-occurrence Matrices (GLCM) and Local Binary Patterns (LBP).

4. Classifier: Classifies the images according to the feature vectors extracted from the feature extraction module. We combined two classifiers, Support Vector Machine (SVM) and Self-Organizing Map (SOM), with the aforementioned feature extraction methods. Our conclusive results are shown in details in section 3 .

5. Density map: Holds the estimated density values for each defined area unit.

6. Archive (Storage): Stores the density maps of a certain area for a certain time interval.

7. Heat Map: Graphically represents the data as a heatmap to indicate the levels of crowd density which we define in (Table I) in section 3. In the heatmap, low levels of service are indicated by a green color, whereas an orange color would indicate a medium level of crowding and the highest level of crowding is shown in red. 


\section{RESULTS/DISCUSSION}

This section summarizes the results of the experiments which were conducted during our study. In general, we experimented with the same image data using two combinations of feature extraction and classification. We conducted tests on two texture based feature extraction methods; GLCM and LBP. In order to classify the levels of crowd occupancy we used an SOM with the GLCM according to Thakare's survey [7] and Rahmalan et al. study [8], and we coupled SVM with the LBP based on Fradi et al. study [9]. The images used were top view $3 \times 6 \mathrm{~m}^{2}$ images (Fig. 4 a) and side view $7 \times 6 \mathrm{~m}^{2}$ images (Fig. 4 b) taken from the Sai area in Al-Masjid Al-Haram. The classification model was largely based on Fruin's standard levels of services (LOS) [10] as shown in the table below (Table I).
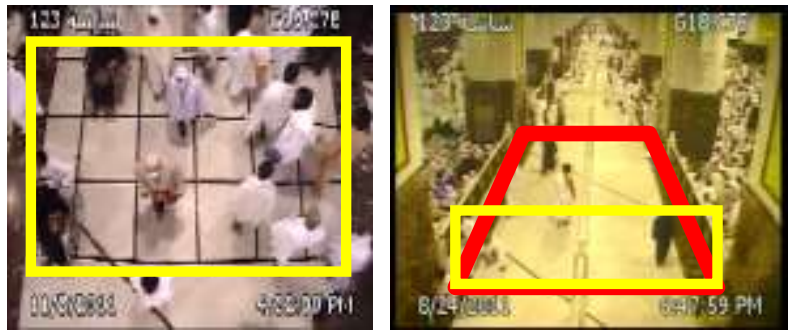

Fig. 4. (a) top view image (b) side view image

TABLE I

OUR LEVEL OF SERVICE CLASSIFICATION

\begin{tabular}{|c|c|}
\hline $\begin{array}{c}\text { Density } \\
\text { (pedestrian/m2) }\end{array}$ & $\begin{array}{c}\text { Our levels of } \\
\text { service }\end{array}$ \\
\hline 0.27 to 0.31 & $\mathrm{~A}($ Low $)$ \\
\hline 0.72 to 1.08 & $\mathrm{~B}$ (Medium) \\
\hline 2.17 to 1.08 & $\mathrm{C}$ (High) \\
\hline
\end{tabular}

The following bar chart (Fig. 5) compares the average accuracy of each of the implemented methods described above. The highest average accuracy was gained by the LBP and SVM at $86.4 \%$ and $87.9 \%$, for top and side view images respectively.

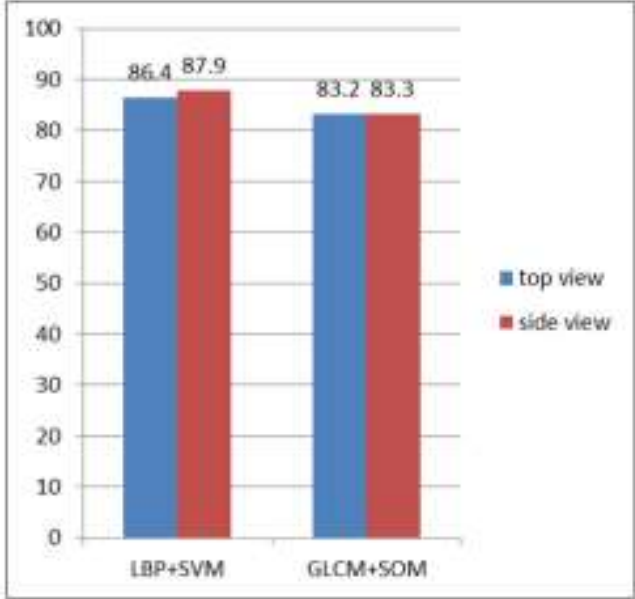

Fig. 5. A comparison between the four experiments

\section{VISUAL REPRESENTATION}

The figures below are screenshots of the system in action. The images (Fig. 7.a, 7.b, 7.c) show the server side of the system estimating the crowd density of a top view of the Sai area in Al-Masjid Al-Haram at three different levels of crowding, high (red), medium (orange), low (green). Fig. 8 shows some of the Android client side interfaces.

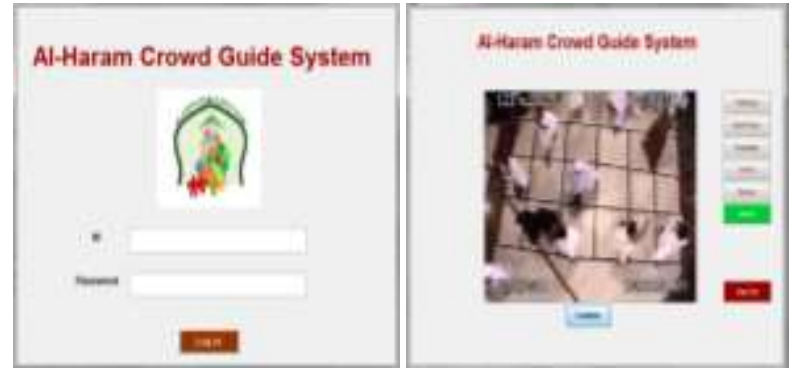

Fig. 6. (a) Administrative Sign-in screen (b) Real-time video feed

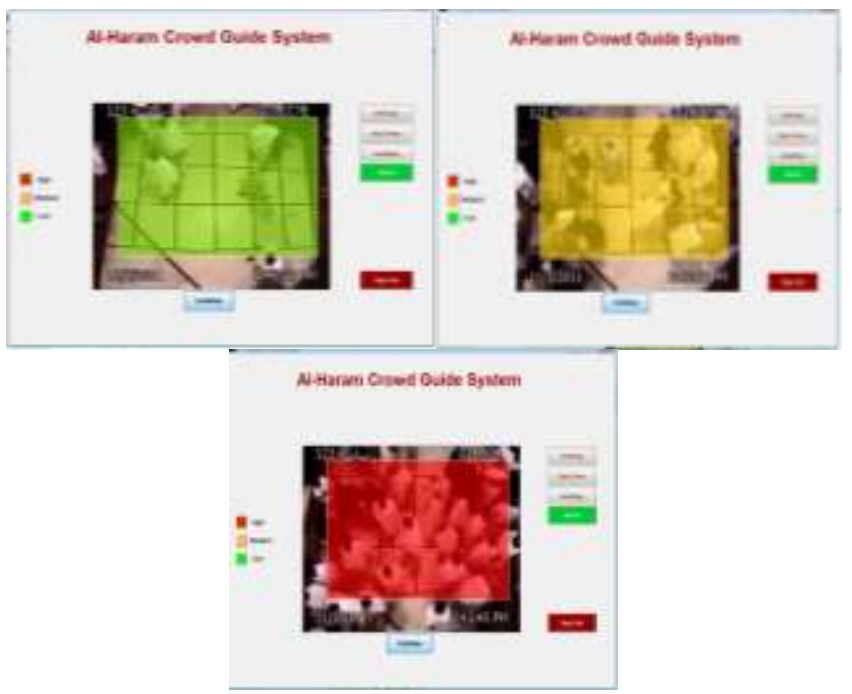

Fig. 7. (a) low density crowding (b) medium density crowding (c) high density crowding

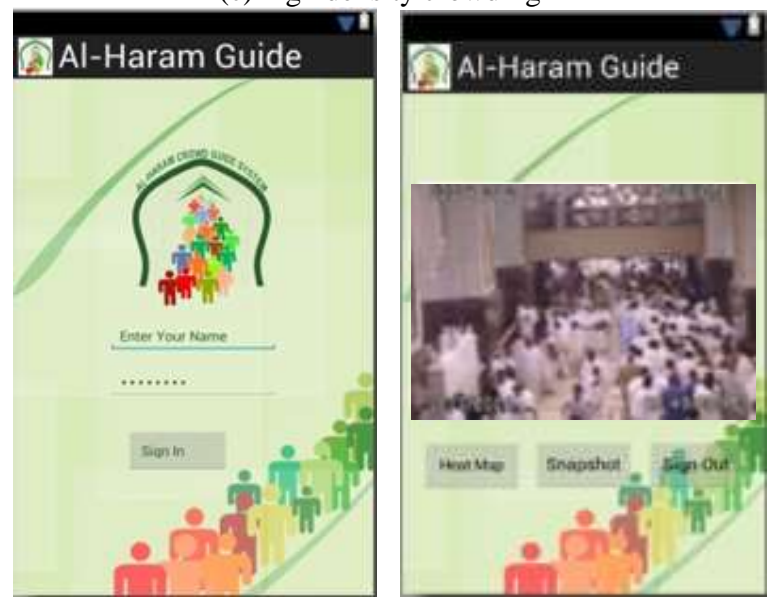

Fig. 8. Interfaces of the client side: (a) Sign in (b) Snapshot of the real view 


\section{CONCLUSION}

At the completion of our study we were able to achieve a fully working prototype of a crowd density estimation system which portrays the levels of density to the user. This paper briefly touches on the experiments conducted using two texture based crowd density estimation methods; LBP and GLCM. From these experiments we confirmed the following finding; a comparison of these methods on both top and side view images showed that the LBP produced the highest overall accuracy when coupled with an SVM reaching an average accuracy of $87.9 \%$,. The future direction of this work would be to include additional features such as estimating crowd flow, in addition to recognizing errant behaviour and dangerous situations. Finally, incorporating crowd density estimation in the field of crowd evacuation is an area of potential study.

\section{ACKNOWLEDGMENT}

We would like to thank Arwa Alharbi, for her help and contribution to the project research. This project was completed by the end of the academic year 2013-2014.

\section{REFERENCES}

[1] B. Zhan, D. N. Monekosso, P. Remagnino, S. A. Velastin, and L.-Q. Xu, "Crowd analysis: a survey," Machine Vision and Applications, vol. 19, pp. 345-357, 2008. https://doi.org/10.1007/s00138-008-0132-4

[2] N. T. Siebel and S. Maybank, "The advisor visual surveillance system," presented at the ECCV 2004 workshop Applications of Computer Vision (ACV), 2004.

[3] S. Velastin, L. Khoudour, B. Lo, J. Sun, and M. Vicencio-Silva, "PRISMATICA: A multi-sensor surveillance system for public transport networks," in Road Transport Information and Control, 2004. RTIC 2004. 12th IEE International Conference on, 2004, pp. 19-25. https://doi.org/10.1049/cp:20040003

[4] London crowd application [Online]. Available: https://itunes.apple.com/gb/app/london-crowd/id545514347?mt=8. Accessed on 7/12/2013.

[5] City of London police application [Online]. Available: http://www.cityoflondon.police.uk/CityPolice/Contact/Smartphoneapp.h tm. Accessed on 7/12/2013.

[6] [Online]:http://www.alriyadh.com/2013/07/27/article855123.html. accessed on 09/12/2013

[7] V. S. Thakare, "Survey On Image Texture Classification Techniques," International Journal of Advancements in Technology, vol. 4, pp. 97-104, 2013.

[8] H. Rahmalan, M. S. Nixon, and J. N. Carter, "On crowd density estimation for surveillance," in Crime and Security, 2006. The Institution of Engineering and Technology Conference on, 2006, pp. 540-545. https://doi.org/10.1049/ic:20060360

[9] H. Fradi, X. Zhao, and J.-L. Dugelay, "Crowd density analysis using subspace learning on local binary pattern," in Multimedia and Expo Workshops (ICMEW), IEEE International Conference, 2013, pp. 1-6. https://doi.org/10.1109/icmew.2013.6618350

[10] Fruin - levels of service [Online]. Available: http://www.gkstill.com/Support/crowd-flow/fruin/LoS.html

Suemayah Eldursi received her B.Sc. (in Computer Science) from Taibah University, Madinah, Saudi Arabia. She previously served at the Department of Computer Sciences, Taibah University, Madinah, Saudi Arabia as a Teaching Assistant and worked online as a Web Developer for EXRX.net. Suemayah is now working as a Full Stack Web Developer in the city of Leicester, England. Her research interests include computer vision, image processing, machine learning and crowd density estimation.
Nada Alamoudi received her B.Sc. (in Computer Science) from Taibah University, Madinah, Saudi Arabia. She is currently a Teacher Assistant at the Department of Computer Science, Community College of Bader, Taibah University, Madinah, Saudi Arabia. She previously served at the Department of Computer Science, College of Engineering and Computer Sciences, Taibah University, Madinah, Saudi Arabia as a Teaching Assistant. Her research interests include visual computing, robotics, machine learning and crowd density estimation.

Fazilah Haron received her B.Sc. (in Computer Science) from the University of Wisconsin-Madison, U.S.A. and her Ph.D. (in Parallel Computing) from the University of Leeds, U.K. She is currently an Associate Professor at the Department of Computer Sciences, Taibah University, Madinah, Saudi Arabia. She previously served at the School of Computer Science, University Sains Malaysia, Penang. Her research interests include modeling and simulation of crowd, parallel processing, image processing, bioinformatics and cloud computing. She has published more than 15 papers specifically, on the research related to crowd at the Masjid Al-Haram and Masjid An-Nabawi.

Faten Aljarbua received her B.Sc. (in Computer Science) from Taibah University, Madinah, Saudi Arabia. Her research interests include computer networks, mobile application developments, artificial intelligence and crowd density estimation.

Ghayda Albakri received her B.Sc. (in Computer Science) from Taibah University, Madinah, Saudi Arabia. She previously served at the Deanship of Information Technology, Taibah University, Madinah, Saudi Arabia as a Technical Support and Customer Service. Ghayda is now working as a Graphic Designer in Madinah, Saudi Arabia. Her research interests include web development, mobile application development, crowd density estimation and artificial intelligence. 